\title{
Introduction to the 37th International Conference on Logic Programming Special Issue II
}

\author{
ALEX BRIK \\ Google Inc., Mountain View, CA, USA \\ (e-mail: abrik@google.com) \\ ANDREA FORMISANO \\ Università di Udine, Udine, Italy \\ (e-mail: andrea.formisano@uniud.it) \\ YANHONG ANNIE LIU \\ Stony Brook University, Stony Brook, New York, USA \\ (e-mail: liu@cs.stonybrook.edu) \\ JOOST VENNEKENS \\ KU Leuven, De Nayer Campus Sint-Katelijne-Waver, Belgium \\ (e-mail: joost.vennekens@kuleuven.be)
}

submitted 08 September 2021; accepted 09 September 2021

This is the second of two issues that contain selected papers of the 37th International Conference on Logic Programming (ICLP 2021), held online from September 20 to 27, 2021. The preceding issue contains a detailed editorial as well as the following 10 selected papers.

\section{Papers from the Main Track:}

- Mario Alviano, Sotiris Batsakis, George Baryannis. Modal Logic S5 Satisfiability in Answer Set Programming.

- Damiano Azzolini, Fabrizio Riguzzi. Optimizing Probabilities in Probabilistic Logic Programs.

- Elena Bellodi, Marco Gavanelli, Riccardo Zese, Evelina Lamma, Fabrizio Riguzzi. Non-ground Abductive Logic Programming with Probabilistic Integrity Constraints.

- Viktor Besin, Markus Hecher, Stefan Woltran. Utilizing Treewidth for Quantitative Reasoning on Epistemic Logic Programs.

- Loris Bozzato, Thomas Eiter, Rafael Kiesel. Reasoning on Multi-Relational Contextual Hierarchies via Answer Set Programming with Algebraic Measures.

- Francesco Calimeri, Marco Manna, Elena Mastria, Maria Concetta Morelli, Simona Perri, Jessica Zangari. I-DLV-sr: A Stream Reasoning System based on I-DLV.

- Angelos Charalambidis, Panos Rondogiannis, Antonis Troumpoukis. A Logical Characterization of the Preferred Models of Logic Programs with Ordered Disjunction.

- Wolfgang Faber, Michael Morak, Lukáš Chrpa. Determining Action Reversibility in STRIPS Using Answer Set and Epistemic Logic Programming. 
- Jorge Fandinno, François Laferrière, Javier Romero, Torsten Schaub, Tran Cao Son. Planning with Incomplete Information in Quantified Answer Set Programming.

- Pascual Julián-Iranzo, Fernando Sáenz-Pérez. Planning for an Efficient Implementation of Hypothetical Bousi Prolog.

This issue contains the remaining 10 selected papers.

\section{Papers from the Main Track:}

- Bishoksan Kafle, Graeme Gange, Peter J. Stuckey, Peter Schachte, Harald Sondergaard. Transformation-Enabled Precondition Inference.

- Tuomo Lehtonen, Johannes P. Wallner, Matti Järvisalo. Harnessing Incremental Answer Set Solving for Reasoning in Assumption-Based Argumentation.

- Vladimir Lifschitz. Here and There with Arithmetic.

- Jukka Pajunen, Tomi Janhunen. Solution Enumeration by Optimality in Answer Set Programming.

- Miguel A. Sanchez-Ordaz, Isabel Garcia-Contreras, Victor Perez-Carrasco, Jose F. Morales, Pedro lopez-Garcia, Manuel V. Hermenegildo. VeriFly: On-the-fly Assertion Checking via Incrementality.

- Kylian Van Dessel, Jo Devriendt, Joost Vennekens. FOLASP: FO(.) as Input Language for Answer Set Solvers.

- Felix Weitkämper. An Asymptotic Analysis of Probabilistic Logic Programming, with Implications for Expressing Projective Families of Distributions.

Papers from the Applications Track:

- Paul S. Brown, Vania Dimitrova, Glen Hart, Anthony G. Cohn, Paulo Moura. Refactoring the Whitby Intelligent Tutoring System for Clean Architecture.

- Carmine Dodaro, Giuseppe Galatà, Andrea Grioni, Marco Maratea, Marco Mochi, Ivan Porro. An ASP-based Solution to the Chemotherapy Treatment Scheduling Problem.

- Müge Fidan, Esra Erdem. Knowledge-Based Stable Roommates Problem: A RealWorld Application.

We wish to thank again the Program Committee members and external reviewers, the other conference organizers, the leadership of Association of Logic Programming, the editorial board of Theory and Practice of Logic Programming and staff of Cambridge University Press, as well as all authors and participants of ICLP 2021 for making the conference a success. 